Psychological Medicine, 2004, 34, 1373-1374. C C 2004 Cambridge University Press

DOI: $10.1017 /$ S0033291704003782 Printed in the United Kingdom

\title{
IN THIS ISSUE
}

This issue features an Invited Review on birth cohort studies in psychiatry, and original articles reporting major follow-up studies of psychiatric disorder, studies of depression, genetic studies, factors associated with disorder in the community, and neuropsychology of schizophrenia.

\section{Birth cohort studies}

In the last 10 years, the coming to fruition of some major birth cohorts has begun to provide data on the early origins of psychiatric disorder of a kind which earlier retrospective studies, depending on potentially biased recall, could only hint at. Colman \& Jones (pp. 1375-1383), in the lead Invited Review, discuss these. We are now in their prime era, when older retrospective methods are increasingly becoming superseded. This and other longitudinal methods have featured increasingly in Psychological Medicine, and are utilized in several studies reported in this issue.

\section{Follow-up studies}

One longitudinal method is the follow-up study. Three papers report long-term outcome findings on psychiatric disorders. Tyrer et al. (pp. 1385-1394) report 12-year outcome of subjects with dysthymia, panic disorder and general anxiety disorder. Only one third were free of a DSM diagnosis at follow-up. Actual diagnoses were unstable over time, supporting Tyrer's view that these are really part of a more general neurotic disorder. Fichter \& Quadflieg (pp. 1395-1406) report 12-year outcome of bulimia nervosa. They find a favourable course, with $70 \%$ of patients free of DSM-IV eating disorder, and very few developing anorexia nervosa. Klump et al. (pp. 1407-1418) make another use of outcome information, to study personality of subjects recovered from eating disorders, and find increased harm avoidance, lower self-directedness and cooperativeness than in normal controls, point to traits underlying the disorder.

Two further follow-up studies concern community subjects. McKenzie et al. (pp. 1419-1430) find Australian 1991 Gulf War veterans more than a decade later have more psychological symptoms than veterans from the same era who were not deployed in the Gulf. Kreicbergs et al. (pp. 1431-1441) report a follow-up at 4-9 years of parents who experienced the death of a child due to malignancy, and a control sample of non-bereaved parents. Although anxiety and depression were elevated up to 6 years, they were not at 7-9 years. Time, evidently, does at least partly heal.

\section{Studies of depression}

A number of papers concern depression. Melartin et al. (pp. 1443-1452), in an 18-month followup study, find both few differences in Axis I or II co-morbidity between melancholic and nonmelancholic depressions on DSM-IV criteria, and considerable switch between melancholia and non-melancholia in subsequent episodes, calling into question the value of the distinction. Majer et al. (pp. 1453-1463) in a short-term follow-up find impaired divided attention associated with non-repsonse to treatment, and risk of relapse. Goodwin et al. (pp. 1465-1474), make use of use a longitudinal birth cohort to show that asthma in adolescence and young adulthood is associated with increased likelihood of depressive and anxiety disorders. Kendler and colleagues (pp. 1475-1482) in a community sample find development of major depression predicted by neuroticism, childhood sexual abuse and recent stressful life events, with sensitivity to life events increased with severity of childhood sexual abuse. In a further, retrospective aetiological study in young women, Hill et al. (pp. 1483-1493) find somewhat different early factors associated with childhood onset and adult onset depression. 
Two studies relate to depression in the elderly. In community subjects aged 60-64 years, Jorm et al. (pp. 1495-1506) confirm earlier studies in finding complaints of poor memory related depression and anxiety, but not on indicators of dementia, including MRI. In elderly homecare patients, McAvay et al. (pp. 1507-1517) find informant report a useful procedure which reveals depression not shown on patient self-report.

\section{Genetic effects}

In Australian twin pairs, Knopik et al. (pp. 1519-1530), confirm substantial genetic influences on alcoholic dependence, but take the field further by showing that this heritability remains, even after controlling for other psychiatric and social risk factors. In a 14-year follow-up of Finnish adoptees, Wahlberg et al. (pp. 1531-1541) find genetic liability for schizophrenic spectrum disorder and communication deviance in adoptive parents both predict future psychiatric disorder, principally in interaction.

\section{Factors associated with disorder in the community}

Weich and colleagues (pp. 1543-1551) examine, in ethnic minorities in a community survey in England, prevalence of so-called common mental disorder, which, in contrast to psychoses, has been little studied in this context. They find only small differences, and particularly no elevated rates in Afro-Caribbeans, who have well-documented elevated rates of schizophrenia. Pevalin \& Ermisch (pp. 1553-1559) examine effects rather than causes of elevated scores on the GHQ, and find in a community survey that poor mental health increases the risk of a cohabitation dissolving, rather than turning into a marriage, and also reduces the likelihood of repartnering after a non-marital cohabitation.

\section{Neuropsychology of schizophrenia}

Three studies report aspects of the neuropsychology of schizophrenia. Knoblich et al. (pp. 15611569) find deficient self-monitoring compared with controls. Birchwood and colleagues (pp. 15711580) find, in patients with auditory hallucinations, that perceptions of low social rank and power are mirrored in voice content and experience. In a prospective longitudinal study, Schiffman et al. (pp. 1581-1586), in another important contribution of longitudinal methods, find that children showing deficits in a role-taking task, related to theory of mind, tend in adulthood to develop schizophrenia spectrum disorder, and also non-psychotic disorder. 